\title{
TECHNICAL DIAGNOSTICS OF MARINE EQUIPMENT WITH PSEUDO-DISCRETE FEATURES
}

\author{
Guixin Fan ${ }^{1)}$, Natalia Nikolova(1),2), Ty Smith' ${ }^{1)}$ Kiril Tenekedjiev ${ }^{1), 2)}$ \\ ${ }^{1)}$ University of Tasmania (Australia) \\ ${ }^{2)}$ Nikola Vaptsarov Naval Academy (Bulgaria)
}

\begin{abstract}
We present a system for technical diagnostics (TD) that can recognize the actual state of marine equipment. A Bayesian classifier is trained to identify the different classes of a piece of equipment, monitored through multiple pseudodiscrete features. Data learning samples can be acquired with direct experiments for each class. The system is capable of merging subjective expert knowledge and data learning samples using pseudo-Bayesian estimates when the parameters of the conditional likelihood for the classes are identified. In the training process, correction is applied to solve numerical problems arising from zero probabilities. The pseudo-discrete features have hybrid nature and combine probabilistic and fuzzy approaches. They combine the ease of extracting subjective expert knowledge typical for the discrete features with the high precision of using the measured data during recognition typical for the continuous features. The domain of each pseudo discrete feature is divided into several main categories of non-overlapping intervals which are described as words by the expert. If a measured feature falls between two consecutive categories it is treated as a linear combination of those categories. The resubstitution performance of the classifier is assessed using an error matrix. A numerical example of a marine diesel generator demonstrates the proposed algorithm in a classification problem with nine different state classes of the generator, monitored through 23 pseudo-discrete features. Data learning samples are acquired with direct experiments for each class. The created TD system has potential applications in other complex engineering systems and may support improvements in marine engineering education and training.

Keywords: fuzzy-probabilistic merging; pseudo-bayesian parameter estimation; learning information, pattern recognition
\end{abstract}

\section{Introduction}

The technical diagnostics (TD) process has been a topic rising in popularity, as industry continues to seek ways to lower spendings on maintenance and minimize system downtime losses. With the aid of reliability engineering, the process of recognizing the working status of any machine or complex system into classes (Koc et al. 2012) has 
been simplified immensely. However, with a complex system or complex piece of machinery, the diagnostic process remains tedious for maintenance personnel. More and more unexperienced personnel, or staff unfamiliar with the workings/operations of such systems become in charge of maintenance and monitoring. Without a good knowledge of the proper workings of such systems, diagnosis of potential faults becomes problematic, since they often face large amount of monitored data without knowing the meaning behind it, or what it indicates.

In this paper, we introduce a TD system using a multi-class classifier, based on pseudo-discrete features (see (Duda et al. 2001) and (Nikolova et al. 2019) for further discussion on pseudo-discrete features). We shall demonstrate the application of the system to recognize the actual state of a hypothetical marine diesel generator in a numerical example. We shall train a Bayesian classifier to identify nine different state classes of the equipment, monitored through 23 pseudo-discrete features. For the learning process, we shall combine subjective expert knowledge and data learning samples using pseudo-Bayesian estimates (Skaggs et al. 1989) when the parameters of the conditional likelihood for the classes are identified. We shall apply epsilon correction in the training process to solve numerical problems arising from zero probabilities.

In what follows, we first present the structure of the technical diagnostics system, as well as the structure of the information we shall utilize for the training of the classifier and the epsilon corrections we shall apply. Then we present the application of the Bayesian classifier to a numerical example for the hypothetical MTU 8V396 marine diesel generator. The last section concludes the paper.

\section{System Description}

Let us analyse a complex system or a system component with several working statuses, categorized into classes. To monitor the system's working status (or class), a vector $\vec{X}$ is introduced as a $d$-dimensional measurement of properties (e.g. temperature, pressure, flowrate, displacement, etc.), where each measured property is a pseudo-discrete feature, represented with $x_{i}, i=1,2,3 \ldots d$, as follows: $\vec{X}=\left(x_{1}, x_{2}, \ldots, x_{d}\right)^{T}$

Therefore, at a given measured property of the component, the posterior probability of the component being in a given class/status can be written with the aid of Bayesian theorem (Ebeling 2010):

$$
P\left(\omega_{k} \mid \vec{X}\right)=\frac{P\left(\omega_{k}\right) P\left(\vec{X} \mid \omega_{k}\right)}{P(\vec{X})}
$$

where

$$
P(\vec{X})=\sum_{k=1}^{c} P\left(\omega_{k}\right) P\left(\vec{X} \mid \omega_{k}\right)
$$


The probabilities of each class $P\left(\omega_{k}\right)$ are known as priors, $P\left(\vec{X} \mid \omega_{k}\right)$ are the conditional likelihoods, and $P(\vec{X})$ is the unconditional likelihood. The working status of the machine/system could be assessed by collecting data from various pseudo-discrete features $x_{i}$. The assessment process would be simple provided that a sufficient, if not abundant, amount of data is available. In practice, however, not every personnel running such complex machineries could make a judgement on the working status based on recorded data.

Take an air compressor as an example. For simplicity, we can define three possible classes for the compressor: normal operation, air leak, and overheating. $\left(\omega_{1}, \omega_{2}\right.$ and $\left.\omega_{3}\right)$ To monitor these classes, we measure four pseudo-discrete features: air pressure, air flowrate, oil pressure and oil flowrate, i.e. $\vec{X}=\left(x_{1}, x_{2}, x_{3}, x_{4}\right)^{T}$.

Measurements from each pseudo-discrete feature are recorded. An operator may have to make a judgement based on the information presented in Table 1. By looking at the numbers presented in Table 1, it is exceedingly difficult for anyone to decide as of which class the compressor is working under. However, the decision making would be much easier if the person is presented with system information as in Table 2.

Table 1. Numerical Data Presentation

\begin{tabular}{|ll|l|}
\hline Air Pressure $\quad \boldsymbol{x}_{1}$ & $200 \mathrm{kPa}$ \\
\hline Air Flowrate $\quad \boldsymbol{x}_{2}$ & $1.5 \mathrm{~kg} / \mathrm{s}$ \\
\hline Oil Pressure $\quad \boldsymbol{x}_{3}$ & $50 \mathrm{kPa}$ \\
\hline Oil Temperature $\boldsymbol{x}_{4}$ & 40 degrees Celsius \\
\hline
\end{tabular}

By looking at the numbers presented in Table 1, it is exceedingly difficult for anyone to decide as of which class the compressor is working under. However, the decision making would be much easier if the person is presented with system information as in Table 2.

Table 2. Pseudo-Discrete Presentation

\begin{tabular}{|l|l|}
\hline Air Pressure $x_{1}$ & Normal \\
\hline Air Flowrate $\quad x_{2}$ & Normal \\
\hline Oil Pressure $\quad x_{3}$ & High \\
\hline Oil Temperature $x_{4}$ & Critical \\
\hline
\end{tabular}

Table 2 presents a much clearer picture of the system's working status. Even a person not familiar with the compressor's normal working condition could tell that the compressor is likely overheating. Therefore, we aim to build a technical 
diagnostic system, using simple pseudo-discrete features that are easy to understand, with the aid of some expert knowledge and learning sample.

\section{Expert Knowledge}

To build a diagnostic system based on Table 2, each pseudo-discrete feature $x_{i}$ is categorized further into pseudo-discretes $\mu_{j, i}$ (meaning the $j^{\text {th }}$ pseudo-discrete of the $i^{\text {th }}$ pseudo-discrete feature), describing the measurement in "levels" such as "too high", "high", "normal", "low" and "too low". The pseudo-discretes are divided using expert knowledge.

Using the same air compressor example, the pseudo-discrete feature oil temperature could be divided into four levels: low, normal, high and critical, with corresponding temperature ranges assigned as shown in Table 3 and Figure 1.

Table 3. Division of pseudo-discretes

for pseudo-discrete feature Oil Temperature

\begin{tabular}{|l|l|}
\hline Low & Below $30^{\circ} \mathrm{C}$ \\
\hline Normal & $40-60^{\circ} \mathrm{C}$ \\
\hline High & $70-90{ }^{\circ} \mathrm{C}$ \\
\hline Critical & Above $100{ }^{\circ} \mathrm{C}$ \\
\hline
\end{tabular}

The expert knowledge provides the level ranges for each pseudo-discrete feature. Such levels are a lot easier to assess than an unexplained/uncategorized data.

Note that the conditional probability of each pseudo-discrete feature can then be represented with $h_{i}$ typical non-overlapping intervals $\left[D_{j}, U_{j}\right]$, which according to (Tenekedjiev et al. 2006) are:

$$
P\left(x_{i} \in\left[D_{j}, U_{j}\right] \mid \omega_{k}\right)=q_{j, i}^{k(x)}
$$

For example, the conditional probability of the air compressor being in third class (overheating), with the fourth pseudo-discrete feature (oil temperature) being in the second pseudo-discrete $h_{2}$ (normal), is:

$$
P\left(x_{4} \in\left[40^{\circ} \mathrm{C}, 60^{\circ} \mathrm{C}\right] \mid \omega_{3}\right)=q_{2,4}^{3}
$$




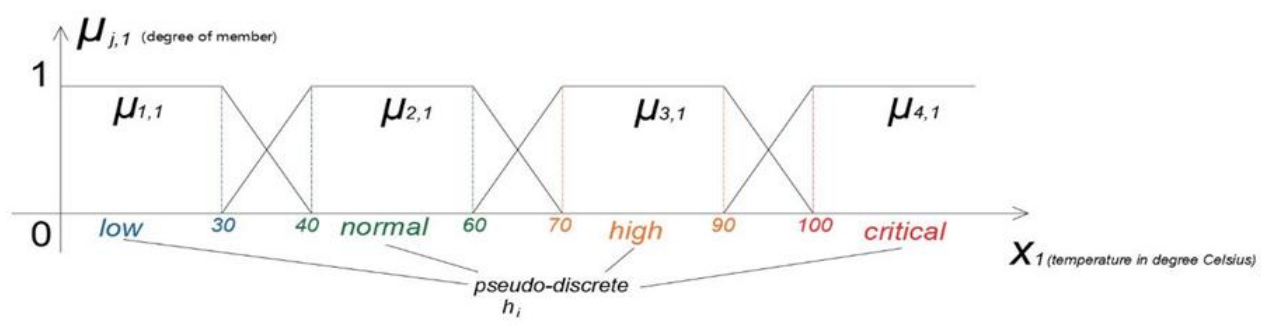

Figure 1. Fuzzy membership Function for Pseudo-discrete feature oil temperature with four pseudo-discretes

After consulting with an expert, the term $q_{j, i}^{k(e)}$ is gained as the reference probability of the $i^{\text {th }}$ pseudo-discrete feature to be in the $j^{\text {th }}$ pseudo-discrete while the system is working under class $k$. We can then build an expert knowledge vector for the $i^{\text {th }}$ pseudo-discrete feature as:

$$
\vec{E}_{i}^{k}=\left(q_{1, i}^{k(e)}, q_{2, i}^{k(e)}, q_{3, i}^{k(e)}, \ldots, q_{h_{i}, i}^{k(e)}\right)
$$

A confidence factor $L_{d}^{k(e)}$ is applied to the expert knowledge. Such factor is a measurement of the expert's accuracy or confidence when assigning probabilities to each pseudo-discrete with a given class. Applying the confidence factor to (4) gives the expert knowledge vector:

$$
E_{k}=\left(\vec{E}_{1}^{k}-L_{1}^{k(e)}, \vec{E}_{2}^{k}-L_{2}^{k(e)}, \ldots, \vec{E}_{d}^{k}-L_{d}^{k(e)}\right)
$$

With each measured property now divided into pseudo-discretes for easier recognition, it is necessary to give the machine a learning sample to study and recognize the class of a system.

\section{Learning Sample}

For each pseudo-discrete feature, $n k$ observations are given to the program for learning and recognition. A vector $\vec{X}_{l}^{k}$ consisting results of all pseudo-discrete features from the $l^{\text {th }}$ observation in class $k$, is then given as:

$$
\vec{X}_{l}^{k}=\left(X_{l, 1}^{k}, X_{l, 2}^{k}, X_{l, 3}^{k} \ldots, X_{l, d}^{k}\right)
$$

For example, in the air compressor case, the vector $\vec{X}_{25}^{3}$ will be: $\vec{X}_{25}^{3}=(101 \mathrm{kPa}$, $1.5 \mathrm{~kg} / \mathrm{s}, 200 \mathrm{kPa}, 88^{\circ} \mathrm{C}$ ) and represents readings from all four pseudo-discrete feature of the $25^{\text {th }}$ observation, when the compressor is overheating. The air pressure reads $101 \mathrm{kPa}$, air flow rate reads $1.5 \mathrm{~kg} / \mathrm{s}$, oil pressure reads $200 \mathrm{kPa}$, and the oil pressure reads $88 \mathrm{C}^{\circ}$. 
The term $q_{j, i}^{k(x)}$ from (3) is calculated using the frequentist definition of probability (Tenekedjiev et al. 2002)

$$
q_{j, i}^{k(x)}=\frac{\sum_{l=1}^{n k} L_{l}^{k} \mu_{j, i}\left(X_{l, i}^{k}\right)}{\sum_{l=1}^{n k} L_{l}^{k}}
$$

with a goodness-of-fit factor $L_{l}^{k}$. The factor shows how well each observation truly represents the corresponding faults or class of a machine (Hald 2007).

\section{Pseudo-discretes}

There is a potential numerical problem with the conditional likelihood term $P\left(\vec{X} \mid \omega_{k}\right)$ in (1). This term is often a small value, and when it is smaller than the machine epsilon $\varepsilon$, it is treated as 0 in any machine language. To solve the stated numerical problem, $P\left(\omega_{k} \mid \vec{X}\right)$ is split into two terms by taking its logarithm:

$$
\ln P\left(\omega_{k} \mid \vec{X}\right)=A_{k}(\vec{X})+B(\vec{X})
$$

The $B(\vec{X})$ part does not depend from the class $k$, whereas the part $A_{k}(\vec{X})$ is different for each class. It is called the discriminant function for class $k$. That name originates from the fact that we can easily identify the class with the greatest posterior probability as the class with the greatest discriminant function (i.e. we can classify the observation $\vec{x}$ using the maximum posterior probability method based only the discriminant functions): $x \in \omega_{k}$ if $A_{k}(\vec{X}) \geq A_{i}(\vec{X})$ for $i=1,2,3, \ldots, c$

Using the discriminant functions allows us to avoid any numerical problems. Although we will never calculate the part $B(\vec{X})$, it is trivial to derive an expression for the posterior probabilities depending only on the discriminant functions:

$$
P\left(\omega_{k} \mid \vec{X}\right)=\frac{1}{\sum_{j=1}^{c} e^{A_{j}(\vec{X})-A_{k}(\vec{X})}}
$$

Taking the logarithm of $P\left(\omega_{k} \mid \vec{X}\right)$ in equation (1) we have:

$$
\ln P\left(\omega_{k} \mid \vec{X}\right)=\ln \left(\frac{P\left(\omega_{k}\right) P\left(\vec{X} \mid \omega_{k}\right)}{P(\vec{X})}\right)=\ln P\left(\omega_{k}\right)+\ln P\left(\vec{X} \mid \omega_{k}\right)-\ln P(\vec{X})
$$

Independent of the class number $k$, it is then recognized that the term $-\ln P(\vec{X})$ 
is to be the term $B(\vec{X})$, and the two terms $\ln P\left(\omega_{k}\right)+\ln P\left(\vec{X} \mid \omega_{k}\right)$ are to be the term $A(\vec{X})$ in $(8)$.

Assuming the measured pseudo-discrete features $x_{i}$ are all independent from each other, then:

$$
P\left(\vec{X} \mid \omega_{k}\right)=\prod_{i=1}^{d} P\left(x_{i} \mid \omega_{k}\right)=P\left(x_{1} \mid \omega_{k}\right) P\left(x_{2} \mid \omega_{k}\right) P\left(x_{3} \mid \omega_{k}\right) \ldots P\left(x_{d} \mid \omega_{k}\right)
$$

The term $P\left(x_{i} \mid \omega_{k}\right)$ is of interest. If the measured feature $X_{i}$ is assumed to be a pseudo-discrete feature with $h_{i}$ pseudo-discretes, then it is possible to represent the theoretical justification of the fuzzy measurements, which leads us back to the setup of pseudo-discrete features and the introduction of expert knowledge section.

\section{Pseudo-Bayesian Estimation}

Combining the learning sample $q_{j, i}^{k(e)}$, and the expert knowledge $q_{j, i}^{k(e)}$, the final probability $q_{j, i}^{k}$ can then be estimated using the frequentist definition of probability with Pseudo-Bayesian Estimation, developed from (7):

$$
q_{j, i}^{k}=\frac{\sum_{l=1}^{n k} L_{l}^{k} q_{j, i}^{k(x)}+50 L_{i}^{k(e)} q_{j, i}^{k(e)}}{\sum_{l=1}^{n k} L_{l}^{k}+50 L_{i}^{k(e)}}
$$

The expert knowledge terms in (12) are applied with an accuracy factor that is subject to change. Here, 50 represents $1 / 50=2 \%$ accuracy factor (Skaggs 1989).

\section{Epsilon Correction}

It is possible that a certain observation would contain no data in certain pseudodiscretes, a.k.a. $q_{j, i}^{k}=0$. In this case, the zero probability is substituted with the machine epsilon $\varepsilon$, while the probabilities from other pseudo-discretes are multiplied by $1-\varepsilon$, so that the sum of probabilities form pseudo-discretes in the same observation still equal to one.

Using the compressor example again, in an observation from the $4^{\text {th }}$ class "Overheating" $(k=4)$, the $4^{\text {th }}$ pseudo-discrete feature "Oil Temperature" $(i=4)$ has 4 pseudo-discretes (low, normal, high, critical $j=4$ ). However, no data falls under the "critical" level. In this case, epsilon correction is performed as shown in Table 4. 
Table 4. Epsilon Correction for $q_{j, 4}^{4}$

\begin{tabular}{|l|l|l|}
\hline Pseudo-Disrete & Original Observation & $\begin{array}{l}\text { Epsilon-Corrected } \\
\text { Observation }\end{array}$ \\
\hline$q_{1,4}^{4}$ & 0.2 & $0.2(1-\varepsilon)$ \\
\hline$q_{2,4}^{4}$ & 0.3 & $0.3(1-\varepsilon)$ \\
\hline$q_{3,4}^{4}$ & 0.5 & $0.5(1-\varepsilon)$ \\
\hline$q_{4,4}^{4}$ & 0 & $\varepsilon$ \\
\hline Sum & 1 & 1 \\
\hline
\end{tabular}

\section{Application on Marine Diesel Generator}

With the established theoretical background, we apply the Bayesian classifier within a numerical example of a hypothetical MTU 8V396 marine diesel generator. The data for our numerical example is obtained from an expert. A total of 9 classes are established, monitored through 23 pseudo-discrete features, i.e., $k=9$, and $i=23$. The classes and features are listed in Table 5 and Table 6.

Table 5. List of Classes for the Marine Diesel Generator Example

\begin{tabular}{|l|l|}
\hline$\omega_{1}$ & Metal Fatigue \\
\hline$\omega_{2}$ & Lost of DC Voltage \\
\hline$\omega_{3}$ & Insufficient Output Frequency \\
\hline$\omega_{4}$ & Single Phase Voltage Drop \\
\hline$\omega_{5}$ & Misalignment \\
\hline$\omega_{6}$ & Faulty Knock in Bore \\
\hline$\omega_{7}$ & Incorrect Air/Fuel Ratio \\
\hline$\omega_{8}$ & Cooler Overheating \\
\hline$\omega_{9}$ & Normal Operation \\
\hline
\end{tabular}

After consulting with an expert, the pseudo-discrete features are classified into 3 to 5 different pseudo-discretes, with ranges $\left[D_{j}, U_{j}\right]$ given to each pseudo-discrete, and expert knowledge $q_{j, i}^{k(e)}$ given to every pseudo-discrete of every pseudodiscrete feature under every class. A learning sample, containing 10 observations in each class are given to the Bayesian classifier for learning and recognition. The confidence factor applied to the expert knowledge is set at $100 \%$ for this analysis.

To demonstrate the parameter estimation methods and to test the performance of the classified the expert also provided 10 pseudo-observations to each class. Some 
of the observations are purposely put out of $\left[D_{j}, U_{j}\right]$ for some pseudo-discrete features to see if the classifier would recognize them as being in a different class.

After recognition, the Bayesian classifier produces an error matrix that can be summarized as follows:

$\omega_{1}: 8$ observations recognized correctly;

2 observations recognized in class 9 ;

$\omega_{2}: 8$ observations recognized correctly;

1 observation recognized in class 8 ;

1 observation recognized in class 9 ;

$\omega_{3}$ : 5 observations recognized correctly;

3 observations recognized in class 4;

2 observations recognized in class 9 ;

$\omega_{4}$ : All observations recognized correctly;

$\omega_{5}: 9$ observations recognized correcly;

1 observation recognized in class 9;

$\omega_{6}$ : 8 observations recognized correctly;

1 observation recognized in class 5 ;

1 observation recognized in class 9;

$\omega_{7}: 9$ observations recognized correctly;

1 observation recognized in class 9;

$\omega_{8}$ : All observations recognized correctly;

$\omega_{9}: 9$ observations recognized correctly;

1 observation recognized in class 8 .

Table 6. List of Pseudo-Discrete Features

for the Marine Diesel Generator Example

\begin{tabular}{|l|l|}
\hline$x_{1}$ & DC Voltage $(\mathrm{V})$ \\
\hline$x_{2}$ & Oil Pressure $(\mathrm{psi})$ \\
\hline$x_{3}$ & Oil Flowrate $(\mathrm{L} / \mathrm{min})$ \\
\hline$x_{4}$ & Oil Temperature $(\mathrm{K})$ \\
\hline$x_{5}$ & Water Temperature $(\mathrm{K})$ \\
\hline$x_{6}$ & Water Flowrate $(\mathrm{L} / \mathrm{min})$ \\
\hline$x_{7}$ & Boost Pressure (bar) \\
\hline$x_{8}$ & Boost Temperature $1(\mathrm{~K})$ \\
\hline$x_{9}$ & Boost Temperature 2 $(\mathrm{K})$ \\
\hline$x_{10}$ & Speed $(\mathrm{rpm})$ \\
\hline$x_{11}$ & Drive-end tri-axel Accelerometer $\mathrm{x}(\mathrm{mm} / \mathrm{s})$ \\
\hline$x_{12}$ & Drive-end tri-axel Accelerometer $\mathrm{y}(\mathrm{mm} / \mathrm{s})$ \\
\hline$x_{13}$ & Drive-end tri-axel Accelerometer $\mathrm{z}(\mathrm{mm} / \mathrm{s})$ \\
\hline
\end{tabular}


Technical Diagnostics of Marine Equipment With...

\begin{tabular}{|l|l|}
\hline$x_{14}$ & Non-drive-end tri-axel Accelerometer $\mathrm{x}(\mathrm{mm} / \mathrm{s})$ \\
\hline$x_{15}$ & Non-drive-end tri-axel Accelerometer y $(\mathrm{mm} / \mathrm{s})$ \\
\hline$x_{16}$ & Non-drive-end tri-axel Accelerometer z $(\mathrm{mm} / \mathrm{s})$ \\
\hline$x_{17}$ & Output Frequency $(\mathrm{Hz})$ \\
\hline$x_{18}$ & Bank A Knock Censor $(\mathrm{mm} / \mathrm{s})$ \\
\hline$x_{19}$ & Bank B Knock Censor $(\mathrm{mm} / \mathrm{s})$ \\
\hline$x_{20}$ & U Single-Phase AC Voltage $(\mathrm{V})$ \\
\hline$x_{21}$ & V Single-Phase AC Voltage $(\mathrm{V})$ \\
\hline$x_{22}$ & W Single-Phase AC Voltage $(\mathrm{V})$ \\
\hline$x_{23}$ & Fule Flowrate $(\mathrm{L} / \mathrm{hr})$ \\
\hline
\end{tabular}

\section{Conclusion}

The process of applying pseudo-discrete features to complex systems could drastically simplify the technical diagnostics process. Our Bayesian classifier was able to recognize the state of the hypothetical generator with few errors on the 120 pseudo-observations provided by the expert. If the given machine is working under a certain class, with sufficient and accurate/confident expert knowledge, the classifier is able to accurately recognized the pattern within the measured pseudodiscrete features.

The use of pseudo-discrete features improves the quality of education in marine engineering, where students need pattern classification in technical diagnostics of marine equipment. These features are easy to use and comprehend. The pattern classification process is more transparent in that way because students can track the diagnostics decisions to their knowledge on how the marine equipment operates.

Future tests of the system should include actual recorded data from running the generator under different conditions, and a few expert knowledge should be made unavailable. The classifier would have to learn from two different scenarios: only learning sample data available with no expert knowledge; only expert knowledge available with no learning sample. In these cases, there is the ability to recognized a pattern by combining known and unknown information and then predict the working class of generator/system using test run data.

\section{REFERENCES}

Duda, R., Hart, P., Stork, D., 2001. Pattern classification. Second Edition. Wiley. Ebeling, C. E., 2010. An Introduction to Reliability and Maintainability Engineering (2nd ed.). Long Grove, Illinois, United States: Waveland Press Inc.

Hald, A., 2007. A history of parametric statistical inference form Bernoulli for Fisher, 1713 to 1935., In: Sources and Studies in the History of Mathematics and Physical Sciences. NY USA: Springer Verlag. 
Koc, L., Mazzuchi, Th., Sarkani, Sh., 2012. A network intrusion detection system based on a Hidden Naïve Bayes multiclass classifier. Expert Systems with Applications 39(18), $13492-13500$.

Nikolova, N., Hirota, K., Kolev, K., Tenekedjiev, K., 2019. Technical diagnostic system in the maintenance of turbomachinery for ammonia synthesis in the process industries. Journal of Loss Prevention in the Process Industries 58, $102-115$.

Skaggs, G., Stevenson, J., 1989. A Comparison of Pseudo-Bayesian and Joint Maximum Likelihood Procedures for Estimating Item Parameters in the Three-Parameter IRT Model. Applied Psychological Measurements. 13(4), 391 - 402.

Tenekedjiev, K., Dimitrakiev, D., Nikolova, N., 2002. Building frequentist distributions of continuous random variables. Machine Mechanics. 47, pp. $164-168$.

Tenekedjiev, K., Kobashikawa, C., Nikolova, N., Hirota, K., 2006. Generic database for hybrid bayesian pattern recognition. Journal of Advanced Computational Intelligence and Intelligent Informatics. 10(3), 419 - 431.

Guixin Fan

https://orcid.org/0000-0002-0334-2271

Australian Maritime College

University of Tasmania

Launceston, TAS, Australia

E-mail: gfan@utas.edu.au

$\triangle$ Natalia Nikolova

ORCID iD: 0000-0001-6160-6282

1Australian Maritime College

University of Tasmania

Launceston, TAS, Australia

Nikola Vaptsarov Naval Academy

Varna, Bulgaria

E-mail: Natalia.Nikolova@utas.edu.au

$\triangle$ Ty Smith

https://orcid.org/0000-0001-6803-7967

Australian Maritime College

University of Tasmania

Launceston, TAS, Australia

E-mail: tasmith9@utas.edu.au ty@nmis.com.au

$\triangle$ Kiril Tenekedjiev

ORCID iD: 0000-0003-3549-0671

Australian Maritime College

University of Tasmania

Launceston, TAS, Australia

Nikola Vaptsarov Naval Academy

Varna, Bulgaria

E-mail: Kiril.Tenekedjiev@utas.edu.au 